\title{
Energy/information dissipation and blood flow in human body
}

\author{
M.Beraia MD. PhD ${ }^{1 *}$, G. Beraia $\mathrm{MD}^{2}$
}

${ }^{1}$ Institute of Clinical Medicine. Tbilisi. Georgia.

${ }^{2}$ Tbilisi State Medical University. Tbilisi. Georgia.

* Corresponding author: M.Beraia, Institute of Clinical Medicine. Tbilisi. Georgia.

Received Date: March 08, 2021; Accepted Date: March 31, 2021; Published Date: April $13,2021$.

Citation: M.Beraia, G. Beraia. (2021) Energy/information dissipation and blood flow in human body. Cardiology Research and Reports. 3(2): Doi: 10.31579/2692-9759/017

Copyright: (C) 2021 M.Beraia. This is an open-access article distributed under the terms of the Creative Commons Attribution License, which permits unrestricted use, distribution, and reproduction in any medium, provided the original author and source are credited.

\section{Abstract:}

The amount of work done to displace blood in systemic arteries and capillaries exceeds the work done by the left ventricle. Besides, at the heartbeat, electromagnetic energy dissipates from the heart to the whole human body.

For the problem study, the dielectric spectroscopy method was used. Ringer's, amino acid solution, and heparinized venous blood were affected by the external electromagnetic oscillations $(100-65000 \mathrm{~Hz}, 1-8 \mathrm{MHz}$.) in 17 healthy individuals. Correlations were noted between the initial and induced signal forms/frequencies according to the impedance of the system.

The electric impulse from the heart initiates an oscillating electric field around the charged cells/particles and an emerging repulsing electromagnetic force, based on the electroacoustic phenomena, promotes the blood flow, in addition to the pulse pressure from the myocardial contraction.

Blood conduces mechanical, electromagnetic waves of different frequencies and transmits energy/information to implement the spontaneous chemical processes in the human body.

Keywords: heart work; blood flow; electromagnetic force; dielectric polarization; erythrocyte; z-potential

\section{Introduction:}

It is accepted that the main factor for blood circulation is the heart. From the law of energy conservation, heart work must be enough for the blood displacement in the vessels, structural changes, and heat production due to the shear flow.

Energy for the blood circulation is expressed as the heart work transferred to the blood via application of the force along with the displacement [1]. Due to the multifaceted nature of the studying problem, some simplifications should be done.

The calculation of the heart work can be successful, in presenting the circulatory system as the compression pump. Disregarding the vicious, inertial forces and the gravity, the work done by the left ventricle must exceed the work of blood displacement in arteries and capillaries.

Under the specified conditions, work for the blood displacement is directly proportional to the pressure $(\mathrm{P})$ and volume $(\mathrm{V})$ of the displaced mass.

$$
\mathrm{W}=\mathrm{P} \times \mathrm{V}=\mathrm{F} \times \mathrm{D}
$$

Amount of the force to the per unit of the area, perpendicular to the surface of the object is a pressure.

$$
\mathrm{P}=-\mathrm{F} / \mathrm{A}
$$

$\mathrm{P}$ - pressure, $\mathrm{F}$ - magnitude of the normal force opposite to the pressure, A - area of the surface on contact. D displacement.
Mean pressure in ascending aorta $100 \mathrm{mmHg}=1.33 .10^{4} \mathrm{~N} / \mathrm{m}^{2}[2]$.

Mean inner diameter of the ascendant aorta $1.5 \mathrm{~cm}$. Mean area of ascending aorta $1.77 \mathrm{~cm}^{2}$.

Volumetric $(75 \mathrm{ml})$ blood mean displacement in ascending aorta in each heart cycle $=42.46 \mathrm{~cm}$.

Force made by the left ventricle is $\mathrm{F}=\mathrm{P} \times \mathrm{A}=1.33 .10^{4} \mathrm{~N} / \mathrm{m}^{2} \times 1.77 .10$ ${ }^{4} \mathrm{~m}^{2}=2.35 \mathrm{~N}$.

Work done by the left ventricle for the blood displacement in the ascending aorta in each heart cycle $\mathrm{W}=2.35 \mathrm{~N} \times 0.42 \mathrm{~m}=0.99 \mathrm{~J}$.

The average capillary hydrostatic pressure $17 \mathrm{mmHg}=22.66 .10^{2} \mathrm{~N} / \mathrm{m}^{2}[3]$.

Mean diameter of the capillary $0.0006 \mathrm{~cm}$. Mean length of the capillary $0.06 \mathrm{~cm}$.

Single capillary volume $=2 \cdot 83 \cdot 10^{-11} \mathrm{~m}^{2} \times 6 \cdot 10^{-4} \mathrm{~m}=16.98 \cdot 10^{-15} \mathrm{~m}^{3}=$ $16.98 .10^{-9} \mathrm{~cm}^{3}$

Quantity of capillaries filled by the $75 \mathrm{ml}$ of blood $=75 \mathrm{~cm}^{3} / 16.98 .10^{-9} \mathrm{~cm}^{3}$ $=$ about $4 \cdot 42 \cdot 10^{9}$ open capillaries.

Hydrostatic pressure force in the single capillary $\mathrm{F}=\mathrm{P} \times \mathrm{A}=$ $22.66 .10^{2} \mathrm{~N} / \mathrm{m}^{2} \times 2.83 .10^{-11} \mathrm{~m}^{2}=64.13 \cdot 10^{-9} \mathrm{~N}$.

Work done for the blood displacement in the singe capillary in each heart cycle $\mathrm{W}=64 \cdot 13 \cdot 10^{-9} \mathrm{~N}$ x $6 \cdot 10^{-4} \mathrm{~m}=79 \cdot 14 \cdot 10^{-12} \mathrm{~J}$. 
Work in blood distribution in all systemic open capillaries (without filtration) in each heart cycle is $\mathrm{W}=79 \cdot 14 \cdot 10^{-12} \mathrm{~J} \times 4.42 \cdot 10^{9}=0.39 \mathrm{~J}$.

In electronic-hydraulic analogy, hydraulic equations can be expressed by the electrical. With this work (W) done on the blood flow in systemic arteries and capillaries can by express as:

$$
\mathrm{W}=\mathrm{Q}^{2} \mathrm{RT}
$$

\section{Q- flow/I-current, R-resistance, T-flow time.}

From the Ohm's law voltage U (pressure- in hydraulic) drop is directly proportional to the current I (Q-flow- in hydraulic) passing through and the resistance $(\mathrm{R})$. $\mathrm{U}=\mathrm{IR}$

Net flow and the flow time in the arteries and capillaries are the same. Pressure drop (so the resistance) in arterial network, up to capillaries $(83 \mathrm{mmHg})$ is 4.9 times higher than that in capillaries $(17 \mathrm{mmHg})$.

Work done in the arterial system must be $0.39 \mathrm{~J}$ x $4.9=1.91 \mathrm{~J}$ and full work done in systemic arteries and capillaries must be about 2.3J. It is higher than that the work done by the left ventricle.

Beside the displacement, structural rearrangement of the blood from the aorta to the systemic capillaries associates with the energetical cost. Womersley's number in fluid biomechanics defines the relation of the transient inertial force to the viscous force in the studied substance. Some typical values of it in different human blood vessels at a heart rate of $2 \mathrm{~Hz}$ are Ascending aorta -13.2, Abdominal Aorta-8; Femoral Artery-3.5; Arterioles - 0.04; Capillaries-0.005 [2]. Changes are happening during the time up to $2 \mathrm{sec}$. and it should be associated with the shear flow (creep/relaxation). At the same time, energy dissipation in turbulence and heat production should be taken into account.

So, the work for the blood displacement in the systemic circulation must be many times more, than the work done by the left ventricle and there should be an additional energy source for this. According to this, it can be shown, that the basic phenomena of plant and animal physiology have an origin of electrical potentials in the cell membrane, and their distribution forms hydraulics - the movement of cytoplasm within a plant or animal cell. Alteration of the potential of charged cell membranes induces their mechanical vibrations together with cell walls, generates acoustic waves in the ultrasound region, and radiates the power [4]. Transmembrane potential can be altered in different parts of the cell, changed with time (ion currents) These processes can be considered as the parametric oscillations, which generate acoustic and electromagnetic oscillations of the ultrasound frequency [5].

Time-varying electric fields can induce several biophysical responses when applied to living cells. One phenomenon of interest is electroporation, also known as electro permeability, in which a rapidly time-varying field increases the permeability of the plasma membrane. Oscillatory electric fields can modulate the conformational states of membrane proteins and have been demonstrated extensively for pumps in the outer plasma membrane [6]. Since the cell membrane is not able to pass current (except in ion channels), it acts as an electrical capacitor. Subjecting membranes to high-voltage electric fields results in their temporary breakdown, resulting in pores that are large enough to allow macromolecules to enter or leave the cell. At frequencies below $10^{4} \mathrm{~Hz}$, the interior of the cell is screened from the applied electric field. At higher frequencies, the electric field is coupled capacitively through the membrane, whereas the counter ions are not sufficiently mobile to screen the interior of the cell so that potential drops are induced across intracellular organelle membranes. Mitochondria, which incorporate a dual membrane structure, play a key role in the energy metabolism of cells. It has been shown that the induced transmembrane potential developed in mitochondrial membranes may affect cellular metabolisms [7].
Another phenomenon is dielectrophoresis - in which a force is exerted on a dielectric particle when it is subjected to a non-uniform electric field. The force depends strongly on the medium and particles' electrical properties, on the shape and size of the particle, as well as on the frequency of the electric field. Consequently, fields of a particular frequency can manipulate particles with great selectivity. This has allowed, for example, the separation of cells or the orientation and manipulation of nanoparticles and nanowires [8].

The characteristic frequencies of oscillation and relaxation motions of the cell envelopes and as a whole be within the range of $10^{3}-10^{6} \mathrm{~Hz}$. The theoretical analysis of natural oscillations of the bilayer lipid membrane, considered as a thin film of a viscous fluid, estimates the lower frequency limit to be $\sim 100 \mathrm{~Hz}$ for the oscillations of the bilayer thickness and $\sim 5 \times 10^{3}$ $\mathrm{Hz}$ for the bending oscillations of the bilayer at an invariable thickness [9].

Cells metabolic and cell-to-cell long-range electromagnetic signaling activities are accompanied by the generation of endogenous electromagnetic fields. Dynamic variations in the length of cytoskeleton microtubules, attached to membrane micro domains were identified as a driving force of cell membrane mechanical vibrations [10].

Low and high-frequency electric fields are involved in the functional alteration of the cell structures. Red blood cells under viscous shear flow are characterized by the tumbling motion - as a rotational oscillation of the entire cell accompanied by tank treading motion. The rotational motion, which is principally due to the cell membrane and occurs around the cell axis, has very low frequencies, of about 1-5 Hz. Erythrocytes oscillate during their tank treading motion with high-frequency oscillations $1.2 \mathrm{MHz}$. This oscillatory motion drastically affects the dielectric and electrical properties of red blood cells [11]

The permittivity of the dielectric is a diagnostic physical property that characterizes the degree of electrical polarization of the material experiences the influence of an external electric field. It is the frequencydependent response of the substance's structure for wave propagation. There is a phase lag between the changes in the substance polarization and the altering external electric field - a measure of the energy loss in a dielectric material through the current conduction - the impedance. Dielectric mechanisms consist of the relaxation and the resonance processes [12.13].

Besides the displacement current, due to the dipolar and ionic polarization, ac signal propagation in the colloid system is associated with the electroacoustic phenomena: Colloid vibration current /potential - an electric signal arises when ultrasound propagates through a heterogeneous fluid such a dispersion or emulsion. Electric sonic amplitude - inverse to the colloid vibration current- in which an acoustic feel arises when an electric field propagates through a heterogeneous fluid [14].

The critical importance of electromagnetic field interactions in biology and medicine has been recognized [15]. Cellular signaling is a complex ballet of molecules interacting and stimulating surrounding proteins, lipids, and ions, resulting in cytoskeletal reorganization, modulation of differentiation, and induction of gene expression. The choreography of events in signaling pathways has been a hot topic in the past few years. The intermolecular reactions should be viewed as a fluid dynamic process in which multiple events may be occurring at the same time [16].

So, the heart work is not enough the providing blood circulation. Herewith, the transmission of electromagnetic energy to the body cells leads to a variety of biophysical processes and can be substantial for the implementation of the energy distribution, as the arterial blood flow. 


\section{Material and methods:}

The dielectric spectroscopy method was used for studying the impedance of the fluid containing Ringer's solution, amino acid solution (AminosolNeo), and heparinized venous blood (Fig1.A). The method measures the frequency response of the system and expressing the energy storage and dissipation by the substance.

The studied fluid was affected by the oscillating electromagnetic field using the frequencies $100-65000 \mathrm{~Hz}, 1-8 \mathrm{MHz}$. Blood was taken from 17 healthy individuals. An induced signal was investigated from the transformer's secondary, silicone catheter coil, filled with the studying fluid. Correlations were noted between the induced signal forms/frequencies in the studied different substances and oscillations of the external magnetic field. Initial electric signal had the square form.

There were correlations of the induced signal forms and frequencies in the studied substances, with the oscillations of the external electromagnetic field. In the substances filling the secondary coil, oscillating electromagnetic field forms ionic and dipolar polarization of the substance and voltage can be measured. Induced signals were looking as RC oscillations (Figure 1.B). In the secondary coil at the low frequencies $(100-300 \mathrm{~Hz})$, it had spike form. At the higher frequency, the substance's capacity has not sufficient time to either full charge during the positive period or fully discharge during the negative period. I have fixed the frequency of the external electromagnetic signals, when the studied substance looked as electrically fully charged (Figure 1B. E). Frequencies for the aminosol $-25000 \mathrm{~Hz}$. saline $-20000 \mathrm{~Hz}$, for the blood $-3000 \mathrm{~Hz}$.

Induced signal frequencies in studied substances are different. In the Ringer's and amino acid solutions induced frequencies were the same as the initial, while in the blood, the difference is significant. Table 1.

\begin{tabular}{|l|l|}
\hline $\begin{array}{l}\text { Signal frequency on the } \\
\text { primary coil }\end{array}$ & $\begin{array}{l}\text { Signal frequency on the } \\
\text { secondary coil }\end{array}$ \\
\hline $100-200 \mathrm{~Hz}$. & $100-200 \mathrm{~Hz}$. \\
\hline $500-1000 \mathrm{~Hz}$. & $400-700 \mathrm{~Hz}$. \\
\hline $3000-5000 \mathrm{~Hz}$. & $2000-3300 \mathrm{~Hz}$. \\
\hline $6000-8000 \mathrm{~Hz}$. & $4000-5000 \mathrm{~Hz}$. \\
\hline $10000-20000 \mathrm{~Hz}$. & $6400-10300 \mathrm{~Hz}$. \\
\hline $40000-65000 \mathrm{~Hz}$. & $20300-17300 \mathrm{~Hz}$. \\
\hline $1-2 \mathrm{MHz}$. & $520-860 \mathrm{KHz}$. \\
\hline $4-8 \mathrm{MHz}$. & $1.1-2.0 \mathrm{MHz}$. \\
\hline
\end{tabular}

Table1: Frequency difference in the primary and secondary coils in the blood study.

Influence of the structure of the different substances in a colloid system of the blood on the specific frequencies may be considered an indirect indication of the existing build-in magnetic field sensing mechanism.

Electric signals with ECG rate 1.1-1.25Hz (65-75 beat/min) cannot induced signals in secondary coil.
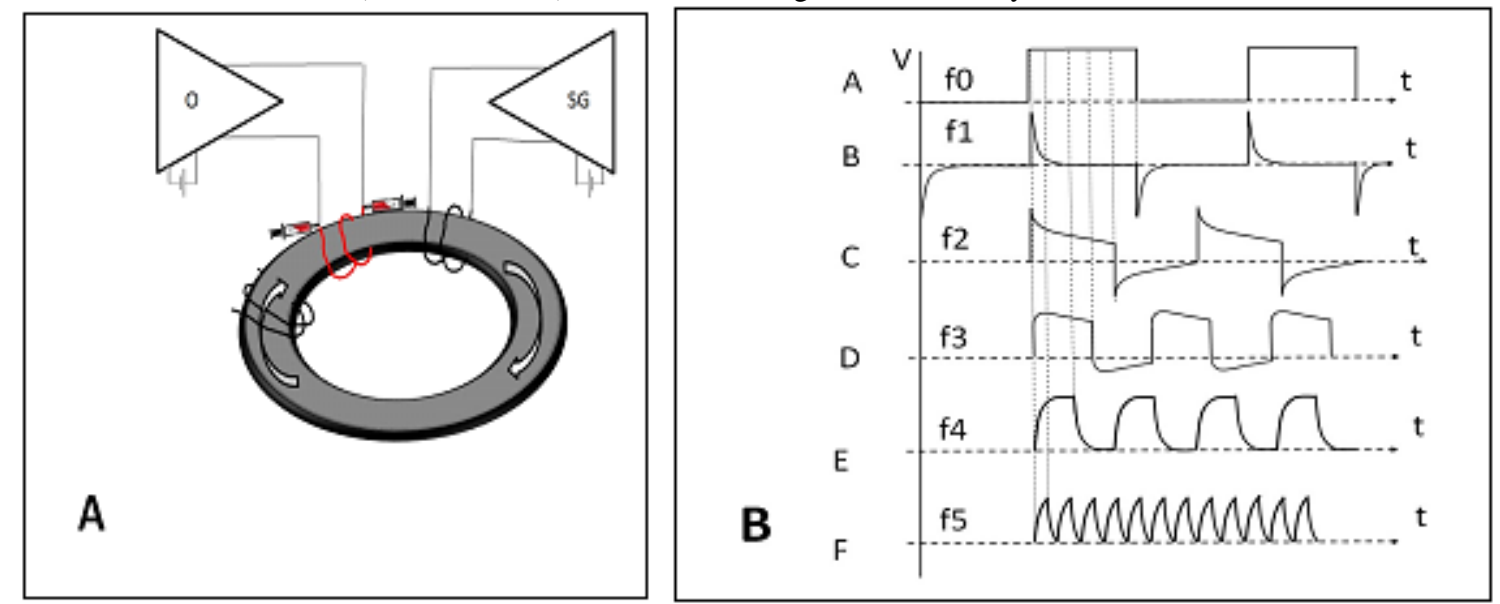

\footnotetext{
Figure 1: A. The signal generator (SG) sends the signals to the high-frequency transformer and induced signals in the secondary coil, registries the oscilloscope $(O)$.

B. Signals in the primary coil are square form (A.) f0=100-65000Hz. Inducting signals look like RC-voltage oscillations.

In the secondary coil at the low frequencies $(f)$, it has spike form $(B)$. The capacity of the investigated substance becomes fully charged quickly and discharges gradually before the next change in the input cycle. The arrival of the falling edge of the input waveform causes the capacitor in the reverse charge giving a negative output spike.

Increasing the frequency, the substance gradually charging and quickly discharges, with the falling edge of the input waveform. Wave uptakes square form (from $C$ to $E$ ).

At the higher frequency, the substance's capacity would not have sufficient time to either fully charge during the positive period or fully discharge during the negative period and the wave takes the triangle form $(F)$.

Substances, taking square shapes in the lower frequency, have a higher electric capacitance. $V$-signal voltage, $t$-time, $(f)$ frequency. f0= $f 1<f 2<f 3<f 4<f 5$.
} 


\section{Discussion:}

Besides the blood flow, electromagnetic energy (ECG) propagates from the heart to the human body. On the infinite initial level of the matter, electromagnetic forces always take part in the displacement of the mass. The electromagnetic field spreads in a vacuum with the speed of the light, however, in solid, or liquids substance, propagation undergoes at a lower velocity. The velocity of the ECG signal is higher than $250 \mathrm{~m} / \mathrm{s}$ [17]. A charged particle produces an electric field by which, exerts on other charged particles. The moving charged particle produces a magnetic field. The magnetic field exerts a force on other moving charges. The force on charges is always perpendicular to the direction of their velocity and therefore only changes the direction of the velocity. Herewith, formed torque is allowed to act through a rotational distance, doing the work [1]. Accelerating of the charges, produce changing electric and magnetic fields. Changing electric fields produce magnetic fields and vice versa. They propagate as the electromagnetic waves transporting energy to the charged particles at the large distance away from the source.

In the bio-colloid system, all cells are electrically polarized. They form membrane potential by the complex interplay between ion transporters and ion channels. Moreover, proteins have electrostatic potential - caused by charged side chains and bound ions - that plays a role in protein folding and stability, enzyme catalysis, or specific protein-protein recognition. In the colloid system, they acquire surface potential. Almost all cell membrane surfaces are intrinsically negatively charged. These negative charges attract opposite charges and create a surface electrical potential, as the $\mathrm{Z}$ potential for the erythrocytes.
Components of the blood include plasma (55\%) and blood cells (45\% most of them are erythrocytes). Plasma contains thousands of different substances, such as proteins, glucose, salts, vitamins, water. These substances are by far lighter than the relatively massive blood cells and their inertia at low frequency is negligible, which affects the conduction by linear transport rather than the oscillation motion. However, the presence of these light materials should not be neglected when studying the electrical properties of the blood because they lead to an important drift-scattered motion alongside an external ac-electric field.

The human body can be considered as a large-volume conductor. During the cardiac cycle, the heart contracts in response to the action potentials, moving along the chambers of the heart. With this, there will be one part of the cardiac tissues, that is depolarized and another part that is at rest or polarized, resulting in the charge separation or dipole. The dipole rotation causes current to flow in the surrounding fluid: At the influence of the external ac-electric field, the micro-particles will rotate by the electrorotation mechanism

Different properties in electro-rotation can be studied by the dielectric spectroscopy method [18]. As it is shown in the difference of the rotational frequencies and/or waveform for the saline, amino acids, and the whole blood (Table 1). Accompanied electroacoustic phenomena/ ultrasound radiation from different cells or even from parts of single-cell, would be independent, i.e. these vibrations will integrally result as an acoustic noise with some possible distribution of frequencies, but the noise of sufficient power is enough for considered phenomena (Figure 2). As the considered processes should be accompanied by electromagnetic radiation on the same frequencies, the registration should be possible by apprehensible electromagnetic devices.

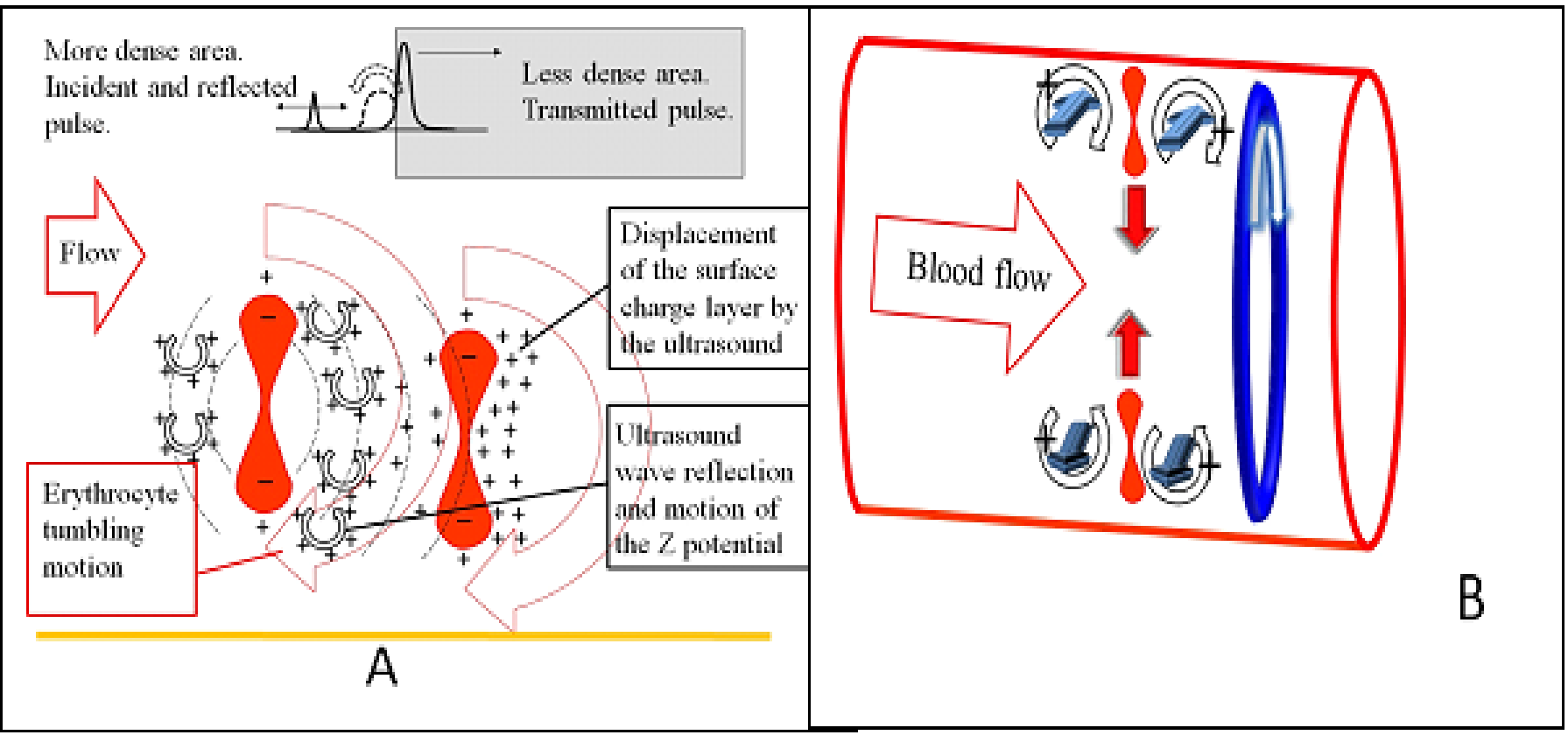

Figure 2: A. Wave at the propagation from the hard to the soft border has no phase change at the reflection, particle rotates in a counterclockwise direction. Erythrocyte rotation contributes to this. Transmitted and reflected waves have the same phase as the initial. The transmitted wave has a higher velocity, than the initial.

The pressure gradient in the ultrasonic wave moves surface charges relative to the erythrocyte. This motion disturbs the double layer that exists at the erythrocyte-fluid interface. Influence of the oscillating electric field to the erythrocyte/charged particles in the colloid system forms electric sonic amplitude-generates ultrasound (A).

$B$. In the artery magnetic fields forming by the rotating erythrocyte dipoles, become a toroidal form (B).

Different authors have demonstrated that the characteristic relaxation frequency of induced micro-dipoles by dielectrophoresis is inversely proportional to the charging and discharging time of the diffuse-layer capacitance in micro fluids which affects both the electrical and mechanical properties of the blood. There is a common frequency of erythrocytes in all the references, $\mathrm{f} \sim 1.2 \mathrm{MHz}$. As this frequency is common and result in form different measurements, several authors, and 
various techniques, one can ask about the nature of this frequency and whether it is an intrinsic property of the erythrocytes [11]. Wave at the reflection from the hard (erythrocyte surface) to the soft border (surrounding substance forming $\mathrm{Z}$ potential), has no phase change and the particle will be rotating in the counterclockwise direction [1]. The transmitted wave has the same phase and higher velocity than the initial. With the above mentioned, oscillation of the $\mathrm{Z}$ potential around the erythrocytes can form a magnetic field and electromagnetic force must be repelling the negatively charged erythrocyte to the central axis of the flow. This may be facilitated by the difference between the dielectric permittivity of the erythrocyte and its environment. Positively charged particles in the surrounding substance will be repelled in opposite direction and this oppositely directed force will be compensated by the vessel wall elastic tension.

Incoming pressure pulse from the heart, destroy equilibrium between the electromagnetic and elastic forces, repulse the erythrocytes in distal direction. Red blood cells take over the plasma, forming the blood flow. This process propagates distally as the domino effect with the accompanying creep and relaxation changes of the viscoelastic substance (Figure 3). Rotational motion of the erythrocytes in the flow more expressed at the Valsalva sinuses/vessel bifurcation area and due to the increasing magnetic field, facilitates the blood flow.

According to this, the arterial pulse wave does not appear on the monitors until 0.16-0.18sec. delay

that the pulse wave velocity in large arteries is $6-9 \mathrm{~m} / \mathrm{sec}$ [19] and location of the large arteries (femoral a.) from the heart is about $1.2 \mathrm{~m}$. the time of the wave propagation from the heart to the large arteries is about 0.13 $0.20 \mathrm{sec}$.

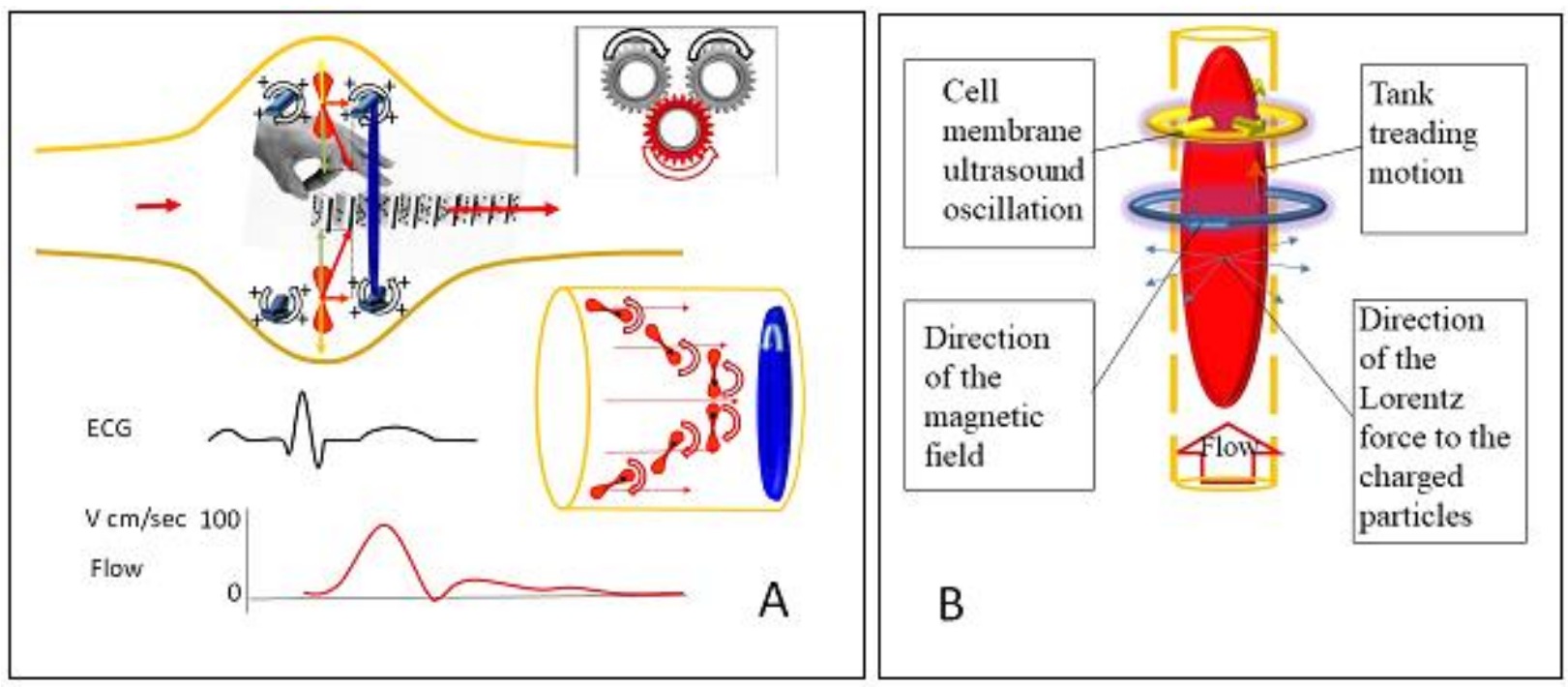

Figure 3: A. Wave at the propagation from the hard to the soft border has no phase change at the reflection, particle rotates in a counterclockwise direction. Erythrocyte rotation contributes to this. Transmitted and reflected waves have the same phase as the initial. The transmitted wave has a higher velocity, than the initial.

Oscillation of the $\mathrm{Z}$ potential around the erythrocytes forms a magnetic field and electromagnetic force repelling the negatively charged erythrocyte to the central axis of the flow. Positively charged particles in the surrounding substance will be repelled in opposite direction and force will be compensated by the vessel wall elastic tension. Incoming pressure pulse from the heart, destroy equilibrium between the electromagnetic and elastic forces, repulse the erythrocytes in distal direction. Red blood cells take over the plasma, forming the blood flow. In the artery, magnetic field, forming by the rotating erythrocyte dipoles, become a toroidal form.

B. In the capillary ultrasound cavitation and electromagnetic forces displaced surface particles in the relationship to the erythrocyte.

Interesting conclusions can be suggested from electrophysiological processes in the heart. The electrical conduction system of the heart transmits signals generated usually by the sinoatrial node. It travels through the right atrium to the atrioventricular node, along with the bundle of His, through bundle branches and Purkinje network to cause contraction of the heart muscle. Bundle branches and Purkinje fibers have high conduction signal velocity $2-4 \mathrm{~m} / \mathrm{sec}$, whereas signal velocity in the ventricular myocardium $0.3-1 \mathrm{~m} / \mathrm{sec}$. Purkinje cells and cardiomyocytes have a short depolarization time $(2 \mathrm{msec})$ and a long refractory period (300msec and $250 \mathrm{msec}$ accordingly). Cardiomyocytes initially depolarize in the intraventricular septum [20]. It forms the oscillating electric field between the opposite walls of the ventricles, facilitates the formation of the electroacoustic, and accompanies electromagnetic phenomena inside the ventricular blood.

Before the blood ejection, pressure force made by the electromagnetic interaction of the charged particles is compensated by the ventricular wall tension. It can be activating the Frank-Starling mechanism. The conducting system is arranged so, that the apices of the ventricles contract before the bases, propelling the blood out of the chambers. At the end of the ECG-QRS signal ventricular wall contraction destroy the equilibrium between the electromagnetic and elastic forces, forms the arterial blood flow like the domino effect, with the accompanying creep and relaxation changes of the viscoelastic substance. Red blood cells take over the plasma, forming the blood flow (Figure 4A.B).

In the electromechanical analogies, the mathematical equations of the rotational mechanical system are compared with the mesh equations of the electrical system. With this, the interaction of the rotational heart dipole to the ECG can be characterized by the torque to voltage analogy. The sequence of ventricular depolarization can be shown by the heart's electrical axis rotation. Elevation of the ECG-QRS is analog to the increasing voltage on the opposite heart ventricular walls, increasing Lorentz force, and shows elevated torque to the erythrocytes and their surface charges (Figure 4C). 

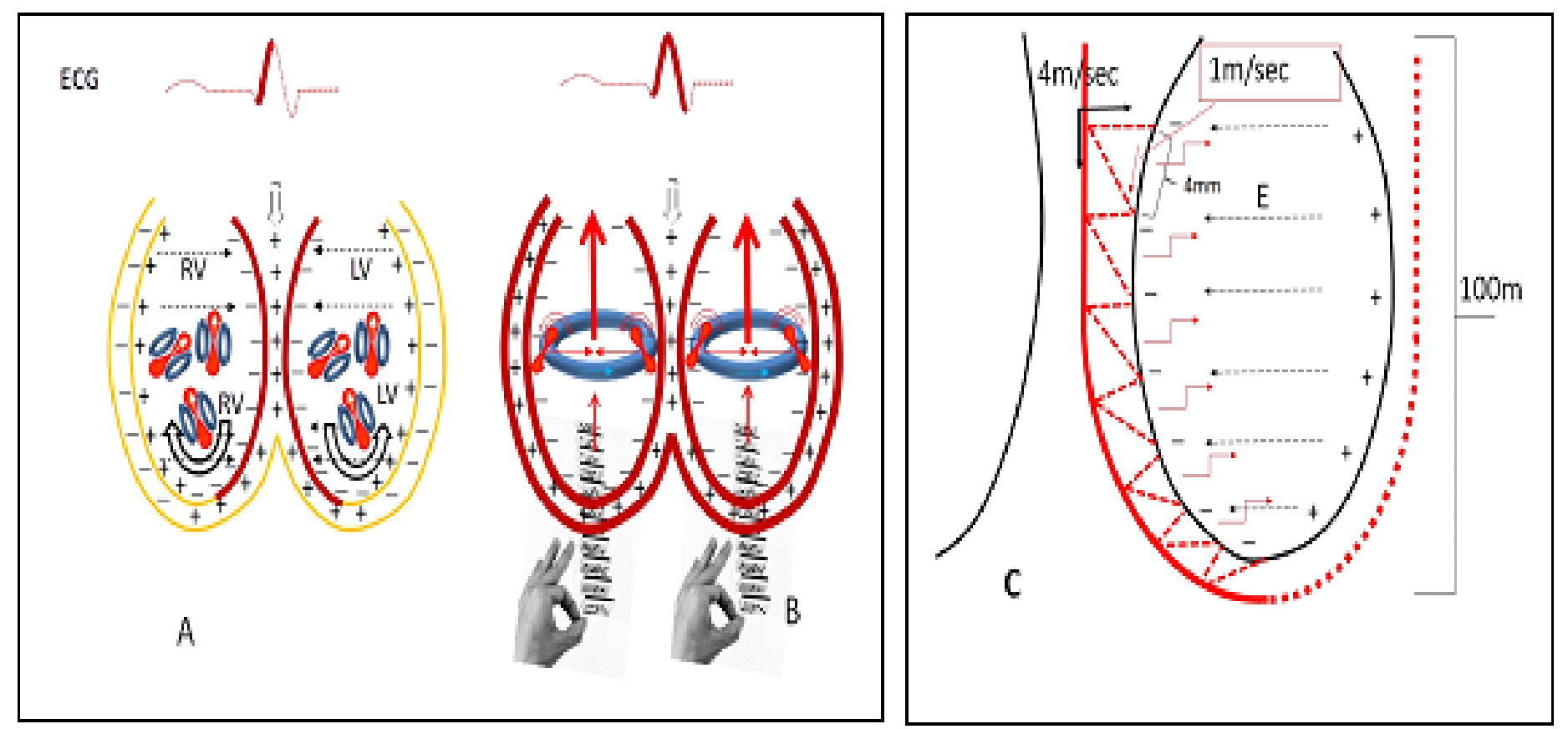

Figure 4: A. Cardiomyocytes initially depolarizing in the intraventricular septum. The oscillating electric field is formed between the septum and free walls of ventricles. Electroacoustic phenomena inside the ventricle form electromagnetic force acting to the charged moving particles. Electromagnetic force compensates by the ventricular wall tension (Isovolumetric contraction).

B. At the end of the electric field oscillation, initially contraction of the apical heart muscles, destroying the delicate balance between the electromagnetic force and wall tension, facilitates propelling the blood out of the chambers in the large arteries, as the domino effect, forming blood flow.

C. The left bundle branch ramifies into the rest of the Purkinje network (dotted red line near the endocardium). Bundle branches and Purkinje fibers have high conduction signal velocity $2-4 \mathrm{~m} / \mathrm{sec}$, whereas signal velocity in the ventricular myocardium $0.3-1 \mathrm{~m} / \mathrm{sec}$. The electric surface charge will be sharply increased by the cardiomyocyte group by the synchronous depolarization by the Purkinje fibers and bundle branches within through each $2 \mathrm{msec}$. along the endocardium with a length of $4 \mathrm{~mm}$.

Due to the sharp depolarization curve and long horizontal plateau of the refractory period in cardiomyocites, the wave oscillation is not sine, but more like square wave. As the electromagnetic field induction is proportional to the acceleration of the charged particle, electric signal can be induces only at the time of depolarization and forming square wave becomes unidirectional.

In the heart conductive system, the left bundle branch is usually a fan-like structure, dividing soon after its origin into anterior and posterior fascicles. These fascicles then further ramify into the rest of the Purkinje network [20]. Conduction is especially rapid through these cells, activating the left side of the interventricular septum, then the apex, finally the base, from endocardium to epicardium. From the physic side, the process of all the cells depolarization cannot be instantaneous. The number of cardiomyocytes in the left human ventricle estimated by design-based stereology reached the maximum value $3.2 \pm 0.75$ billion cells [21]. An endocardial surface negative charge will be sharply increased by the cardiomyocyte group in about the synphase depolarization by the Purkinje fibers, along the bundle branches within through each $2 \mathrm{msec}$. along the endocardium with a length of about $4 \mathrm{~mm}$. In this period signal will be summing and can be depolarized up to 65 million cells. Due to the refractory period of the cardiomyocytes, the Purkinje fiber can be depolarizing only the next nearest group of the muscle cells.

Signal frequency for the cardiomyocyte depolarizations ( $\mathrm{T}=2 \mathrm{msec}$ ) $500 \mathrm{~Hz}$. Time propagation of the impulses in the ventricular conduction system is about $100 \mathrm{msec}$. During this period about 50 myocardial cell groups, ruling by the Purkinje bundles, can be depolarized in each ventricle.

Electric signal from the cardiomyocyte depolarization, transmitted to the blood volume, looks like the voltage oscillation in RC circuit, as you see on the in the graph (Fig.1F). With the increasing of the electric field, the adding charge from the cell depolarization can be increases the amplitude and frequency of the electroacoustic oscillations - up to $2 \mathrm{MHz}$. Signal propagation in the heart conductive system accompanies the rising amplitude of the electroacoustic phenomena and forms the amplitude/frequency modulated electromagnetic wave, express as the ECG.

Displacement current, providing by the heart and the blood, facilitates the electromagnetic energy transport to all cells due to the electronegativity of the oxygen inside the mitochondria.

Energy transfer in universe proceeds in waveform. In the human body it increases the system entropy, implements spontaneous chemical reactions, motion of the substances and the heat formation. Heart represents the setting generator for the modulated carrier signal in $\mathrm{KHz}$ "noise", while electroacoustic phenomena induced from the red blood cells, carry out retranslations of the electromagnetic oscillations in $\mathrm{MHz}$ frequency, provide mass transport in the whole body cells. The ultrasound oscillations accompanying the electroacoustic phenomena can propagate into the existing acoustic guides through the blood vessels and extracellular spaces.

The amount of the energy carried out by the wave is related to their frequency and amplitude: the higher the frequency/the amplitude, the 
more energy there is. The purpose of the carrier frequency is to transmit the information through space as an electromagnetic wave. Modulation of the carrier frequency carries out the transport of the information, for ruling the different cell activities all over the body.

In the signal, modulations take part in different areas of the heart and the substances, solved in the blood. With the interaction of the electromagnetic wave packets with the different chemical structures, diffraction/interference of the wave spectral dissociation can form. Later it leads to the transformation into the wave polarization, and the phase shift [22]. Formed dielectric, dipolar, and ionic polarization of the substance can modify the initial electromagnetic field (Fig1B. A-F). It looks like the information carriers are the molecular structures of the body and blood.

The speed of electromagnetic waves is a property of the "medium" of propagation and is associated with the permittivity of the substance. The velocity of the ECG is at least $25000 \mathrm{~cm} / \mathrm{sec}$ and generated oscillation frequency from the electroacoustic phenomena $1-2 \mathrm{Mhz}$. length of the electromagnetic wave must be $0.1-0.2 \mathrm{~mm}$. It corresponds to the length of the cell structures.

Cell membranes can be extracting the information from the incoming signal. Working as the RC- low/high pass filters, they make integration/differentiation for the electric impulses, modifying input signal. Dielectric dispersion/permittivity of the substance has a function of frequency [23] and acts on metabolic cell activity.

In cellular respiration, the presence of a proton pump creates a transmembrane charge gradient between the two sides of the inner mitochondrial membrane and is the main tool for the energy (ATP) production. Accumulation of the charges also done on the cell/plasma membranes by the external electromagnetic oscillations, at the signal propagation.

Remarkable susceptibility of ion channels to exogenous weak magnetic fields tuned to specific ion resonance frequencies, observed in numerous experiments, indicates of existing build-in magnetic field sensing mechanism employed by living cells routinely for ion traffic regulation and corporative operation.

The point of view that the living system consists of energy and information is commonly accepted. The metabolism of the organism is in essence a cost of information processing. Information handling is considered the most essential feature of the living system. Energy and information components are acting upon and within the living system on the level of microphysical events through their additive values of negative and positive entropy. Mass, energy, and information are not only categories describing, but also additive operative values on the microlevel of physical phenomena. Consideration of the examples of the function of a biological membrane and its models, calculations of experimental equivalents of information negentropy, and lowering the energy cost of biological reactions during onto- and phylogenesis lead us to the conclusion that lowering the energy cost of biological reactions decisive in the emergence of the living system and its biological evolution [24].

While the many questions stay opened for the discussion, the next advance in medicine will be to discern and apply electromagnetic signaling parameters acting to promote wellness of the normal biological processes, with the decreasing reliance on the pharmaceuticals. Investigation of the information basis in pathophysiological processes must be performing by a group of scientists with the adapted laboratory. The author is open to cooperation.

\section{Conclusion:}

The electric impulse from the heart initiates an oscillating electric field around the charged cells/particles and an emerging repulsing electromagnetic force, based on the electroacoustic phenomena, promotes the blood flow, in addition to the pulse pressure from the myocardial contraction.

Blood conduces mechanical, electromagnetic waves of different frequencies and transmits energy/information to implement the spontaneous chemical processes in the human body.

\section{References:}

1. David Halliday, Robert Resnick, Jeral Walker JohnWiley \& sons (2011) Fundamentals of Physics.

2. T.J.Pedley. Cambridge University Press. (1980) The Fluid Mechanics of Large Blood Vessels.

3. Yosef Levenbrown DO, Andrew Thomas Costarino MD, MSCE. (2019) Nephrology and Fluid/electrolyte Physiology (Third Edition) Edema.

4. Mark E.Perlman and Galina M. Rubinstein. (2006) Racah Institute of Physics. Hebrew University of Jerusalem. Ultrasound Vibrations of Plant Cell Membranes: water lift in trees, electrical phenomena.

5. M. E. Perel'man, G. M. Rubinstein. Bull. Acad. Sc. Georgian SSR. (1982) Ultrasound vibrations of plant cells membranes: water lift in trees, electrical phenomena. 107, 393.

6. Vijayanand Vajrala James R. Claycomb Hugo Sanabria John H. Miller Jr. (2008) Department of Physics and Texas Center for Superconductivity, University of Houston, Houston, Texas; Department of Mathematics and Physics, Houston Baptist University, Houston, Texas; and University of Texas Health Science Center, Houston, Texas. Biophysical Journal. Effects of Oscillatory Electric Fields on Internal Membranes: An Analytical Model. Volume 94.

7. H. Potter. Review. Curr. Protoc. Immunol. (2001) Transfection by Electroporation.

8. Yehia H. Ghallab, Waeb Badaway. (2004) Sensing Methods for Dielectrophoresis Phenomenon: From Bulky Instruments to Labon-a-Chip. IEEC. Circuits and Systems Magazine 4(3):5-15.

9. V. L. Kononenko and J. K. Shimkus. Membr. Cell Biol. (2000) Spontaneous and Forced Oscillations of Cell Membrane of Normal Human Erythrocytes: Absence of Resonance Frequencies in a Range of 0.03-500 Hz.Vol.14 (3), pp. 367-382

10. Cells Membrane Vibrations as a Mechanism of Endogenous ELF Magnetic Field Generation in Biosystems. Alex Axelrod.

11. S. Abdalla. (2011) Effect of erythrocytes oscillations on dielectric properties of human diabetic-blood. AIP Advances 1, 012104 .

12. L D Landau L. P. Pitaevskii E.M. Lifshitz. 2-nd edition- Elsevier (1984) Electrodinamics of continuous media.

13. Yoshihito Hayashi, Marc-Aurèle Brun, Kenzo Machida and Masayuki Nagasawa. Anal. Chem. (2015) Principles of Dielectric Blood Coagulometry as a Comprehensive Coagulation Test. 87, 19, 10072-10079.

14. Studies in interface science. Chapter 5. Electroacoustic theory. Volume 15. Elsevier. 2002

15. T. Taghian D.A. Narmoneva, A. B. Kogan. J.R. Soc. (2015) Interface. Modulation of cell function by electric field: a high resolution analysis. 12(107).

16. Approaches to Studying Cellular Signaling: A Primer for Morphologists. Kathy Kay Hartford Svoboda, Wende R. Reenstra. Anat Rec. (2002) ; 269(2): 123-139.

17. Jan Gierałtowski Working Group for Cardiovascular Physics, Faculty of Physics, Warsaw University of Technology. Sixth Cardiology Meets Physics \& Mathematics (2015) How fast does the ECG signal propagate within the body. Teodor Buchner, At Zakopane, Volume: VI. 
18. Kremer, Friedrich, Schönhals, Andreas (2003) Broadband Dielectric Spectroscopy.

19. Tânia Pereira, Carlos Correia, and João Cardoso. J Med Biol Eng. (2015) Novel Methods for Pulse Wave Velocity Measurement. 35(5): 555-565.

20. Lie and Janse. (1978) The Conduction System of the Heart. Structure, Function and Clinical Implications Wellens.

21. Enikő Lázár, Hesham A. Sadek, and Olaf Bergmann. Eur. Heart J. (2017) Cardiomyocyte renewal in the human heart: insights from the fall-out .38(30): 2333-2342.

22. Electronic materials. Principles and applied science. DielectricsInsulators. 1st edition. Yuriy Poplavko. (2018) Elsevier.

23. Tim Hanshaw, Kaitlyn I Franz, Martha Migliacio. (2018) Real Analog: An Introduction to Electrical Circuits.

24. A.T. Pol. University of Miami and Biotech International Inc., Miami, FL 33124, U.S.A. Computers Math. (1990) Life: Energy - Information relationship within material systems. 1.General outline of the concept. Applic. Vol. 20, No. 4-6, pp. 269-285. 